\title{
Maternal nanomaterial exposure: a double threat to maternal uterine health and fetal development?
}

\begin{abstract}
"The potential applications associated with engineered nanomaterials ... are seemingly limitless, particularly in the broad disciplines of biomedical therapeutics and diagnostics, or 'theranostics'."
\end{abstract}

Keywords: engineered nanomaterial $\bullet$ fetal development $\bullet$ microcirculation - nanotoxicology $\bullet$ pregnancy $\bullet$ theranostics

The potential applications associated with engineered nanomaterials (ENMs) are seemingly limitless, particularly in the broad disciplines of biomedical therapeutics and diagnostics, or 'theranostics' [1]. The National Nanotechnology Initiative has invested a considerable amount of resources, research and infrastructure for material development at dimensions less than $100 \mathrm{~nm}$ [2]. Materials manufactured at the nanoscale express unique properties and characteristics that differ from those of their larger counterparts of the same chemical composition. Furthermore, the toxicities and physiochemical properties of these ENMs are distinctly different, and thus are not well understood [3]. Moreover, novel ENMs and ENM properties are generated faster than their toxicities can be determined. Federal resources have been allocated to generate research and development of commercial products and medical technologies within the fields of drug delivery and imaging. To a lesser extent, these resources also support investigations into the toxicokinetics of these novel materials $[2,4]$.

For good reason, most early nanotoxicology research has focused almost exclusively on pulmonary exposures within a young, healthy, male model. Our studies on microvascular dysfunction subsequent to ENM inhalation is of no exception. While this approach has yielded important descriptive and mechanistic information, the increased use of ENMs in novel biomedical and consumer products inevitably leads to increased occupational, environmental and domestic exposures to a variety of ENMs that are both intentional and unintentional. Perhaps some of the applications with the greatest potential to improve human health are those that require the intentional introduction of ENMs to the body. Such ENM exposure routes would no longer be limited to the lungs and would include ingestion, transdermal and injections (intravenous or other). Potential applications under development include drug delivery, high-resolution imaging, preventative measures (antioxidants) and implantable devices. The shear breadth of these applications mandates that we considerably widen our exposure models.

The fetomaternal relationship during gestation is a unique, dynamic and complex physiological system. The term 'milieu', coined by Claude Bernard, or homeostatic environment is often used to describe different compartments, conditions and components associated with a given physiological function. At the outset, maternal health and homeostasis is paramount for a successful gestational outcome; therefore, uterine adaptation to pregnancy (e.g., growth and pressure regulation) or the uterine milieu must be first considered. Incorporated in the gestational uterine milieu is placental development, a transient organ with significant influence over fetal development. In addition to exchange of nutrients and wastes, the placenta serves as an endocrine organ to both the maternal and fetal circulations [5]. Lastly, the intrauterine environment where the fetus develops, or the fetal milieu, must be established and maintained to promote appropriate growth and development. Any dysfunction within the coordinated

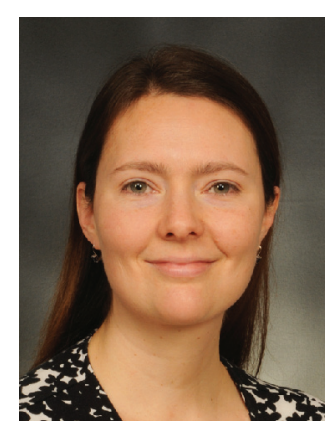

Phoebe A Stapleton Center for Cardiovascular \& Respiratory Sciences, West Virginia University School of Medicine, Morgantown, WV, USA and

Department of Physiology \& Pharmacology, West Virginia University School of Medicine, Morgantown, WV, USA

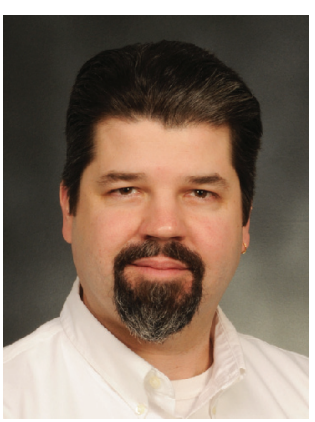

Timothy R Nurkiewicz Author for correspondence: Center for Cardiovascular \& Respiratory Sciences, West Virginia University School of Medicine, Morgantown, WV, USA and Department of Physiology \& Pharmacology, West Virginia University School of Medicine, Morgantown, WV, USA tnurkiewicz@hsc.wvu.edu

Future : Medicine part of 
exchange of hormones, nutrients and wastes during gestation may lead to devastating fetal consequences. Therefore, regulation of maternal homeostasis and the fetal milieu are highly susceptible to a variety of external influences or exposures. Currently, our understanding of ENM exposure in these regards is quite poor. To date, few studies have explored the consequences of maternal ENM exposure during pregnancy [6-8].

Failure to recognize the consequences of ENM exposure during gestation may lead to untoward outcomes for generations. While it would be easy to label nanomaterial theranostics as contraindicated during pregnancy, the potential benefits within the field of obstetric theranostics may be immense. For example, applications of immediate interest to human health may include: fetal imaging, assessment of high-risk pregnancies and early pharmacological interventions. These promising advancements, while exciting and novel, can only reach their full potential if the toxicity of a given ENM, and its terms are first properly understood in all regards.

\section{Importance of characterization}

To properly address the health effects and toxicological consequences of maternal ENM exposure, accurate material characterization of each material is paramount. Common criteria include size, elemental composition, valence, shape and functional groups to adequately address reproducibility and biologic effects. The increasing potential of biomedical use only serves to necessitate these criteria further. ENM hold great potential in the development of personalized and directed medication, specifically as we explore the field of 'theranostic nanomedicine' [1]; however, use of poorly characterized materials within a pregnant patient may directly affect fetal health, thus impairing fetal maturation and reducing theranostic impact.

\section{"Gestational nanotoxicology is a severely understudied area ripe with potential biomedical applications."}

\section{Pregnancy, the microcirculation \& ENMs}

Rapid, yet intricate, maternal vascular adaptation and regulation are crucial for a successful pregnancy. While many systems and organs are ultimately responsible for fetal health and development during gestation, the microcirculation (arterioles, capillaries and venuoles) is fundamentally responsible for proper tissue perfusion in all regards. Local and systemic signaling cascades are responsible for microvascular adjustments (dilation and constriction) to appropriately distribute blood and regulate the high maternal arterial pressures that penetrate the intrauterine tissues. This coordination also prevents areas of ischemia while establishing the delicate nutrient/ waste exchange sink at the capillary level. While uterine pressure distribution and the placental milieu are interdependent, two variables that jointly influence the fetal environment, the consequence of disrupting either due to ENM exposure would probably be an untoward fetal outcome.

Healthy uterine and uteroplacental perfusion that develop during gestation are achieved through rapid structural vascular remodeling described as 'hypertrophic', where the luminal area and wall surface area of a vessel are enlarged without any change to wall thickness [9-11]. In addition, vessel length and microvascular density are significantly increased during gestation to further support fetal growth [10]. Any interruptions to this delicate development and remodeling may lead to disturbances in fetal health and/or unsuccessful gestation.

Alterations to the placental milieu with regard to structure/function (vascular or metabolic) may significantly affect the maternal-fetal coordination of growth and development. When considering direct ENM interaction or translocation, nanomaterials would need to pass through two fetal cell layers to be transferred from the maternal to the fetal circulation within mammals of hemochorial placentation (including humans and rodents) [11]. Given that nanomaterials deposited in the lung have been shown to translocate systemically to a variety of tissues [12-14], it is quite likely that ENM migration to the fetus will occur after maternal exposure. Such nanomaterial translocation could greatly impair embryogenesis [15] or imbed in developing fetal tissues, leading to lifelong consequences. The 'Barker hypothesis' suggests that disturbances in maternal health during gestation greatly impact fetal development, and creates the foundation for adult disease [16]. Traditionally, the development of various maternal pathologies (gestational diabetes [17] or intrauterine growth restriction [18]) has been regarded as the origin of secondary impacts on fetal health. Recently, we have reported that maternal ENM exposure adversely influences litter/pup health [6]. This is consistent with the Barker hypothesis from a nonpathologic perspective. Clearly, more rigorous toxicological investigations are necessary.

Fetal consequences associated with maternal ENM exposure may be derived from indirect (alterations to the intrauterine milieu) or direct (ENM translocation to the fetal compartment) exposure. These consequences may range widely from low success rates, low birth weights, genetic conditions and tetragenic effects to lifelong, disease-based outcomes. The underlying mechanisms may also range from genetic interruptions [8] to metabolic insufficiencies [18]. At this time, the fetal effects of maternal ENM exposure have only 
recently been reported. In these rodent studies, liver cellular signaling, inflammation and metabolism pathways were all affected in the fetal offspring of carbon black exposure instilled into the pregnant dam [8]. Fetal microvascular dysfunction has been reported after maternal $\mathrm{TiO}_{2}$ inhalation during gestation [6]. This also appears to delay postnatal growth [19]. Owing to this limited understanding, future research must expand to identify all fetal consequences and reveal the underlying mechanisms.

\section{Conclusion}

Gestational nanotoxicology is a severely understudied area ripe with potential biomedical applications. These areas may include novel pharmacological therapies aimed to reduce intrauterine growth restriction, reduce birth weights of gestational diabetes patients or the development of fetal contrasting agents for earlier diagnostic potential. In order for nanotechnology to benefit the needs of maternal-fetal health, several immediate needs

\section{References}

1 Chen X, Gambhir SS, Cheon J. Theranostic nanomedicine. Acc. Chem. Res. 44(10), 841 (2011).

2 National Science and Technology Council; Committee on Technology; Subcommittee on Nanoscale Science Engineering and Technology. National Nanotechnology Initiative Strategic Plan. National Nanotechnology Coordination Office, VA, USA (2014).

3 NTP Nanotechnology Safety Initiative. National Toxicology Program Fact Sheet. National Toxicology Program, NC, USA (2006).

4 Borm PJ, Robbins D, Haubold S et al. The potential risks of nanomaterials: a review carried out for ECETOC. Part Fibre Toxicol. 3, 11 (2006).

5 Gude NM, Roberts CT, Kalionis B, King RG. Growth and function of the normal human placenta. Thromb. Res. 114(5-6), 397-407 (2004).

6 Stapleton PA, Minarchick VC, Yi J, Engels K, McBride CR, Nurkiewicz TR. Maternal engineered nanomaterial exposure and fetal microvascular function: does the Barker hypothesis apply? Am. J. Obstet. Gynecol. 209(3), 227-211 (2013).

7 Hougaard KS, Jackson P, Kyjovska ZO et al. Effects of lung exposure to carbon nanotubes on female fertility and pregnancy. A study in mice. Reprod. Toxicol. 41, 86-97 (2013).

8 Jackson P, Hougaard KS, Vogel U et al. Exposure of pregnant mice to carbon black by intratracheal instillation: toxicogenomic effects in dams and offspring. Mutat. Res. 745(1-2), 73-83 (2012).

9 Mulvany MJ, Baumbach GL, Aalkjaer C et al. Vascular remodeling. Hypertension 28(3), 505-506 (1996). must be prioritized. First, specific fetomaternal health challenges and sensitivities must be established. Second, the limitations of existing therapies and/or diagnostics in these regards must be identified. Third, ENM characteristics that overcome these limitations must be sequentially verified in silica, in vitro and in vivo. Finally, all toxicological considerations in these regards must be fully understood. Failure to properly define these needs will prevent and/or delay the full potential that nanotechnology may offer to maternal-fetal health outcomes.

\section{Financial \& competing interests disclosure}

This work was supported in part by NIH grants R01-ES015022 (TRN) and F32-ES023435 (PAS), and NSF-1003907 (TRN). The authors have no other relevant affiliations or financial involvement with any organization or entity with a financial interest in or financial conflict with the subject matter or materials discussed in the manuscript apart from those disclosed.

No writing assistance was utilized in the production of this manuscript.

Osol G, Moore LG. Maternal uterine vascular remodeling during pregnancy. Microcirculation 24, 58-71 (2013).

11 Osol G, Mandala M. Maternal uterine vascular remodeling during pregnancy. Physiology (Bethesda) 24, 58-71 (2009).

12 Stapleton PA, Minarchick VC, Cumpston AM et al. Impairment of coronary arteriolar endothelium-dependent dilation after multi-walled carbon nanotube inhalation: a time-course study. Int. J. Mol. Sci. 13, 13781-13803 (2012).

13 Mercer RR, Scabilloni JF, Hubbs AF et al. Extrapulmonary transport of MWCNT following inhalation exposure. Part Fibre Toxicol. 10(1), 38 (2013).

14 Yokel RA, Tseng MT, Dan M et al. Biodistribution and biopersistence of ceria engineered nanomaterials: size dependence. Nanomedicine 9(3), 398-407 (2013).

15 Kulvietis V, Zalgeviciene V, Didziapetriene J, Rotomskis R. Transport of nanoparticles through the placental barrier. Tohoku J. Exp. Med. 225(4), 225-234 (2011).

16 Barker DJ. Intrauterine programming of adult disease. Mol. Med. Today 1(9), 418-423 (1995).

17 Hiersch L, Yogev Y. Impact of gestational hyperglycemia on maternal and child health. Curr. Opin. Clin. Nutr. Metab. Care 17(3), 255-260 (2014).

18 Simmons RA. Developmental origins of diabetes: the role of oxidative stress. Best Pract. Res. Clin. Endocrinol. Metab. 26(5), 701-708 (2012).

19 Blum JL, Xiong JQ, Hoffman C, Zelikoff JT. Cadmium associated with inhaled cadmium oxide nanoparticles impacts fetal and neonatal development and growth. Toxicol. Sci. 126(2), 478-486 (2012). 